\title{
A metal foreign body pulmonary embolism
}

\section{J Joubert, MB ChB, Mmed Rad D \\ Worcester Radiology, Worcester, W Cape}

\section{Background}

Retention of a foreign body in the heart or great vessels may be due to direct penetrating injury or embolism. In a series by Jiang Chun- $\mathrm{Li}^{1}{ }^{1}$ retained foreign bodies were usually the result of penetrating cardiovascular injuries secondary to gunshot or stab wounds. The clinical manifestations of such patients vary from asymptomatic to haemodynamic instability.

\section{Case history}

The patient had a history of penetrating abdominal trauma; 6 years before, a metal projectile (a piece of wire) entered his pelvis during a farming accident. X-rays on 6 May 2003 confirmed a metal fragment in the pelvis. Six years later, on 4 April 2009, the patient was again involved in a farming accident and sustained facial bone fractures, contusion of the right lung, a right pneumothorax, as well as sacral and pelvic fractures. X-rays taken on 4 April 2009 revealed the same metal object as

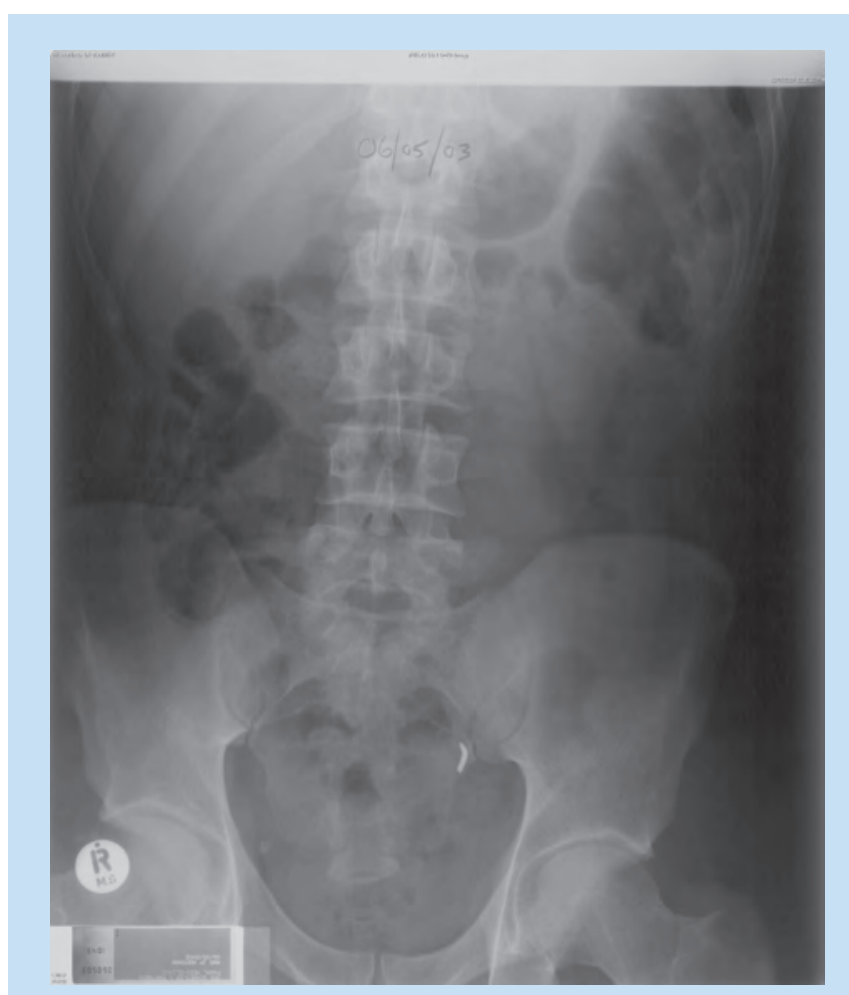

Fig. 1. X-ray of 2003 shows a metal foreign body in the pelvis.

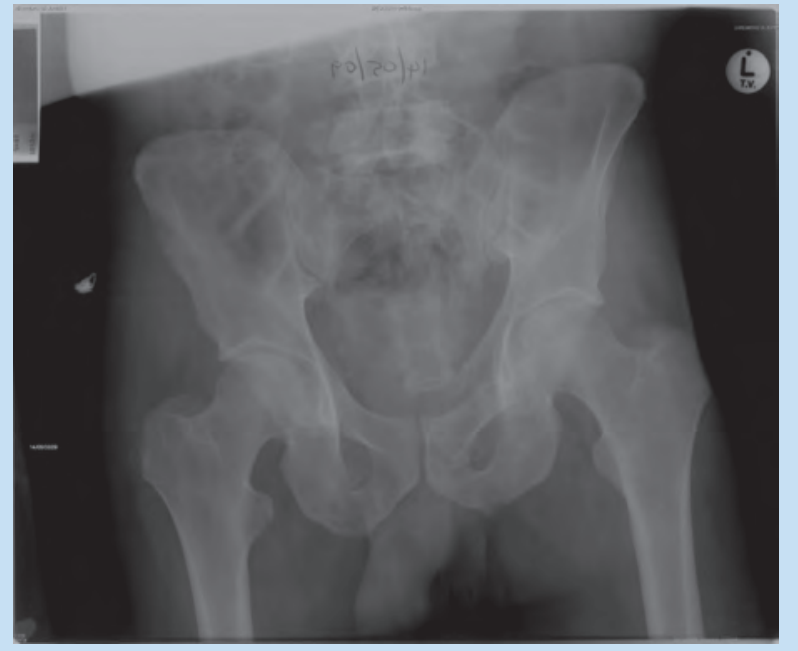

Fig. 2. X-ray of April 2009 shows no foreign body in the pelvis.

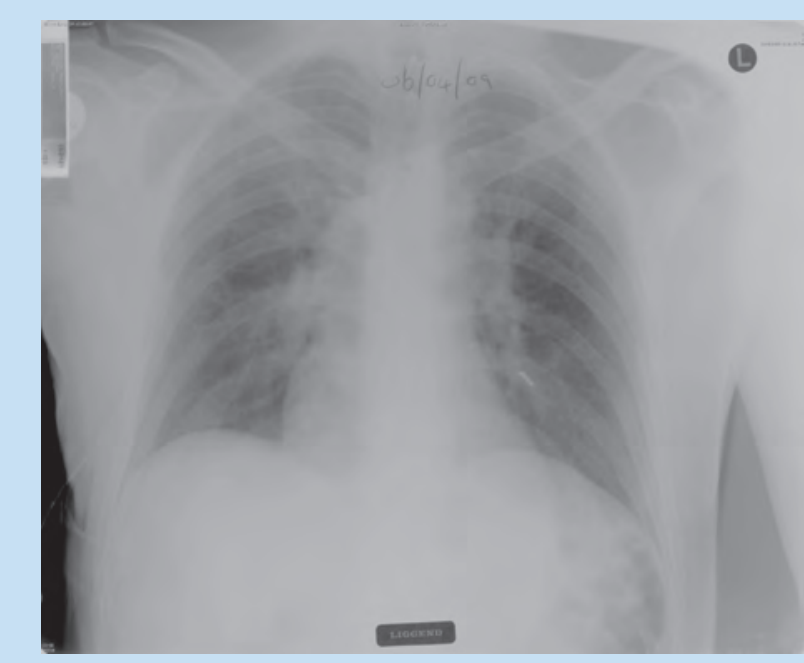

Fig. 3. The same metal object that was visible previously in the pelvis can now be seen in the left lower lung region.
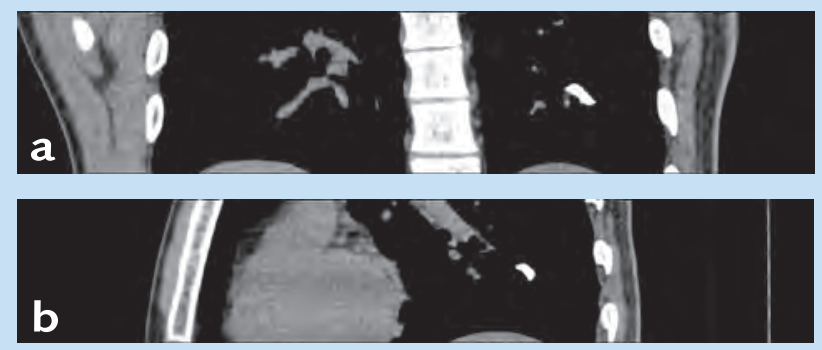

Figs $4 a$ and $4 b$. Uncontrasted (coronal and sagittal reconstructions) CT scans of the chest demonstrate the metal foreign object in a pulmonary artery branch to the left lower lobe. 


\section{CASE REPORT}

seen before, but this time located in the left lung. A CT scan of the chest confirmed a piece of metal in a pulmonary artery to the left lower lobe.

\section{Discussion}

The foreign body in the pelvis of our patient most probably migrated from the pelvis to the inferior vena cava and from there to the right atrium and right ventricle, from where it entered the left pulmonary artery to the pulmonary circulation loop. This could have happened in the 6 years between the X-rays or possibly during the trauma. Foreign bodies can usually be diagnosed with conventional radiology, but a clinical history is also of value. Conventional X-rays are sufficient to make a correct diagnosis of a metal foreign body, but a Multislice CT scan is indicated for precise location. Management options include surgical or conservative therapy (Dr Theo Franken, personal communication, 22 May 2009), and should be individualised after the risk evaluation of removal of the foreign body.

\section{Conclusion}

This rare case demonstrates migration of a metal missile from the pelvis to the left pulmonary artery after a penetrating abdominal injury.

1. Jiang Chun-li, Gu Tian-xiang, Wang Chun. Surgical treatment of post traumatic foreign bodies in the heart and great vessels. Chin Med J 2006; 119(23): 2018-2020.

2. Vagvolgyi A, Vadasz P, Heiler Z, Cseko A. Delayed pulmonary embolism caused by a wandering bullet after abdominal shooting injury - case report. Presented at the 17th European Conference on General Thoracic Surgery, 31 May - 3 June 2009, Krakow, Poland. 